\title{
Blucher
}

Blucher Proceedings

Cuba e Brasil no Século XXI (CBS21)

Inovação e Desenvolvimento Sócioeconômico Sustentável

\section{El Puerto de Mariel: los impactos en los procesos de desarrollo.}

\author{
Dr. José Manuel Mateo Rodríguez ${ }^{1}$ y Dra. Regina Oliveira ${ }^{2}$
}

\begin{abstract}
Introducción
Desde la preparación de la primera reunión CBS21 en el 2012 (Alianza Académica CUBA y BRASIL en el Siglo XXI), quedó evidente que uno de los momentos fundamentales en la formulación de una sociedad del conocimiento en Cuba y Brasil era la temática ambiental y territorial.

En la sociedad del conocimiento, el medio ambiente y el territorio emergen como objetos preferenciales del quehacer cognitivo. Ello es, porque constituyen fuentes de recursos y servicios, siendo un espacio físico, fuente de sentimientos estéticos y culturales, laboratorio genético. Es decir, además del valor de uso, se consideran cada vez más como valor de intercambio. Conocer el medio ambiente y el territorio es un requisito fundamental para la construcción de cualquier proceso de sociedad del conocimiento.

Desde la primera reunión celebrada en La Habana, quedaron claras dos direcciones en que se precisaba enfilar los esfuerzos científicos:

- La temática de la educación para la sostenibilidad, como base para la implementación de procesos de desarrollo. Ello se formuló a través del diseño y la creación de la Red EGASOS - Educación Superior y gestión ambiental para la sostenibilidad en la sociedad del conocimiento en Cuba y Brasil.

\footnotetext{
${ }^{1}$ Universidad de La Habana, Academia de Ciencias de Cuba (ACC), Cuba. E-mail: mateo@geo.uh.cu.

${ }^{2}$ Universidade Estadual de Campinas (UNICAMP), Campinas, SP, Brasil. E-mail: reginacoliveira@ige.unicamp.br.
} 
- La temática del Ordenamiento ambiental y territorial, formulándose la posibilidad de ejecutar proyectos concretos de investigación aplicada y básica orientada.

Teniendo en cuenta, la necesidad de orientar la colaboración entre Cuba y Brasil en los proyectos de alcance estratégico entre ambos países, se consideró pertinente prestar atención preferencial a la experiencia de la implantación del puerto de Mariel, mega obra construida con apoyo y la participación del Brasil. Para ello se ha partido de la experiencia en los últimos años del Brasil de privilegiar la implantación de polos de desarrollo en puertos de nuevo tipo, como estrategia fundamental del uso, la ocupación y la apropiación de los territorios y sus recursos y de inserción en la geopolítica y la geo economía mundial. Además, se ha tenido en cuenta toda la experiencia teórico conceptual y empírica brasileira en la esfera de la incidencia de las grandes mega obras en el desarrollo regional y territorial.

En el presente artículo, los autores se dedican a analizar las necesidades de conocimiento que surgen en cuanto a la construcción del puerto del Mariel, en relación con los efectos y consecuencias básicamente de orden ambiental y territorial que tendrá dicha obra. Gran parte del referencial teórico se ha tomado de trabajos especializados sobre la temática del desarrollo (Mateo, 2012) y experiencias brasileiras sobre el impacto e os puertos a nivel territorial. (Lima et al, 2004; Monie, 2012).

\section{El Puerto de Mariel}

Mariel es una "bahía de bolsa" situada a 45 kilómetros al oeste de La Habana, con un canal de entrada de unos 300-500 metros que abre a una bahía de 5-6 kilómetros de largo y 3-5 kilómetros de ancho con una profundidad de hasta 10 metros.

Tomando como punto de partida la creación del puerto, y las instalaciones a el asociadas, se ha creado por disposiciones gubernamentales La Zona Especial de Desarrollo Mariel (ZED) ubicada en la cuenca marítima del norte de la parte occidental del país se enmarca en el municipio del mismo nombre, en la provincia de Artemisa, en el cual se localizan 32 asentamientos, de ellos 8 urbanos y 24 rurales con una población de 41,658 habitantes, de ellas se encuentran en estado laboral 26,504 habitantes.

La ciudad de Mariel, con una población de 43,000 habitantes, se encuentra en su extremo noreste. El municipio cuenta con 11,836 viviendas, fondo habitacional que no cumple con las necesidades de la población. En el puerto se prevé en una primera etapa que debe concluir en el 2014, con la construcción de un muelle de $700 \mathrm{~m}$, permitirá la entrada a buques de mayor calado. Además se trabaja en la 
puesta en práctica de infraestructuras que comunicara esta zona con la Capital del país, entre las que se encuentra la ampliación del ferrocarril y la construcción de una autopista hacia el Mariel, que entronca con la Autopista La Habana - Pinar del Rio.

Igualmente, se aumentará el volumen de carga pues tendrá espacio para tres millones de contenedores de carga, cifra muy superior a la capacidad actual del puerto de La Habana. Se implantarán plantas de ensamblaje y diversos tipos de industrias que podrían producir bienes para América Latina y Europa. El gobierno brasileño, financia $\$($ USD)640 millones del costo total de $\$($ USD)900 millones.

Se pretende el desarrollo de cuatro grandes actividades en lo fundamental:

1. La construcción y explotación de la Terminal de Contenedores Mariel como negocio líder.

2. La creación del Centro para la Logística a las Petroleras en la Zona Económica Exclusiva Cubana del Golfo de México.

3. El desarrollo de una Zona Económica articulada con las dos anteriores con el aprovechamiento de la sinergia que se debe propiciar entre los tres conjuntos y un desempeño con clase mundial.

4. El desarrollo de una Zona Agropecuaria que permita alcanzar altos niveles de producción de alimentos para satisfacer las demandas internas del territorio e incluso del entorno regional.

De acuerdo al decreto ley Número 313 emitido por el Consejo de Estado de la República de Cuba (23 de septiembre de 2013) se ha creado la "Zona Especial de Desarrollo Mariel" (ZED), que tiene un emplazamiento de 465,4 kilómetros cuadrados, cuyos objetivos son:

- Contribuir al desarrollo nacional.

- Generar exportaciones y promover la sustitución de importaciones.

- Propiciar la transferencia de tecnología de avanzada y know how y de habilidades referidas a la gerencia de negocios.

- Atraer la inversión extranjera.

- Generar nuevas fuentes de empleo y de financiamiento a largo plazo.

- Propiciar la sostenibilidad ambiental.

- Desarrollar la infraestructura necesaria para contribuir al progreso económico. 
- Crear un sistema logístico que permita altos niveles de eficiencia en los procesos de importación, exportación y distribución.

- Estimular el establecimiento de empresas nacionales o extranjeras; y

- Garantizar su articulación con el resto de la economía.

Se dispone en dicha resolución regímenes especiales dirigidos a garantizar el funcionamiento óptimo aduanero, laboral, financiero, y logístico para los usuarios que se establezcan en la zona. Los mismos tendrán derecho a transferir al exterior en moneda libremente convertible sin pago de impuestos, las utilidades netas o dividendos que obtengan provenientes de sus actividades, así como repatriar el capital invertido.

\section{Importancia de la Zona de Desarrollo Especial del Mariel (ZED)}

En el mundo en general, y en Brasil en particular, se está imponiendo una nueva estrategia en el desarrollo, y en la organización espacial. Se trata de que los puertos construidos desde hace más de un siglo, vinculados a grandes ciudades, están prácticamente saturados. Las grandes urbes, están cumpliendo otras funciones, y la función portuaria o se está reduciendo, re funcionalizando y especializando o está siendo sustituida, por una función turístico marítima, o incluso se están abandonando.

Los nuevos puertos que se construyen se edifican a cierta distancia de los antiguos puertos, no se vinculan a distancias cortas a grandes urbes, y constituyen polos de industrialización, y de intercambio de mercancías. Son en realidad polos de desarrollo, que se gestionan como figuras especiales (Zonas francas, zonas especiales de desarrollo etc.). En no pocas ocasiones, el puerto en sí, es el polo de atracción de una zona de desarrollo regional, que traspasa la influencia más cercana del propio puerto.

Brasil, construye en Mariel el mayor puerto del Caribe, región que enfrenta un impetuoso desarrollo transnacional, vinculado con la ampliación del canal de Panamá, la ampliación o construcción de otros puertos en diversos puntos de la región, y la posibilidad de creación de otros canales inter oceánicos través de la América Central. Brasil tiene varias experiencias en la construcción de polos de desarrollo portuarios. Entre ellos se destacan el puerto de Suape en Pernambuco, el de Pecem en Ceará y el de Açu en la región de Campos, al norte del Estado de Rio de Janeiro.

La experiencia de Suape, ha sido positiva. Se ha convertido en el polo de mayor desarrollo actualmente en el Brasil. Pero, junto con esa expansión, el crecimiento 
económico ha desbordado en parte la capacidad de planificación, y ocurren situaciones, que según algunos está conduciendo a una bancarrota ambiental y social. Para Cuba, es importante tener en cuenta las experiencias positivas y negativas de Suape y otros puertos brasileiros, no solo para prever posibles anomalías, sino para diseñar una organización espacial y ambiental racional y equilibrada. Aquí es necesario el conocimiento sobre las características regionales, su dinámica, sus reacciones a los impactos, para diseñar estrategias efectivas en relación con el desarrollo territorial.

Los impactos de la implantación de polos de desarrollo portuario, se manifiestan en toda la organización espacial, no solo del país involucrado, sino incluso a nivel global y macro regional. Constituyen en lo fundamental, momentos desencadenadores del desarrollo territorial, visto no solo en el sentido de la sinergia que crean los procesos de maximización física y crecimiento económico, sino en el sentido de incremento de la organización, la complejidad y la optimización en cuanto a los soportes espaciales y la movilización de recursos y servicios, que en definitiva se manifiestan en un amplio entorno espacial. Para potenciar los efectos y consecuencias positivas y minimizar las negativas, que pueden analizarse al menos en tres niveles, (macro regional/ nacional/ local) es necesario su conocimiento. A continuación se expondrán los posibles impactos en los mencionados niveles.

\section{Impactos a Nivel Global y Macro Regional del Puerto de Mariel}

El Puerto del Mariel constituirá una zona de transbordo y distribución de mercancías en un eje comercial dinamizado que conectaría al Extremo Oriente y Asia con la costa este de los Estados Unidos, Europa, Meso y Suramérica. Se trata de una oportunidad. La remodelación del Canal de Panamá para permitir barcos mayores con cargas hasta tres veces superiores, coloca al Caribe en el vértice de un intenso tráfico comercial y en un lugar privilegiado solo comparable a los tiempos de las Carreras de Indias. Pero se trata de una oportunidad muy disputada en la que intervienen - en la práctica o solo en proyectos - puertos caribeños (Kingston, Ponce, Punta Caucedo en Santo Domingo, Freeport en Barbados y Mariel en Cuba), y puertos centroamericanos y norteamericanos de la costa este.

El puerto de Mariel está ubicado en la parte septentrional del llamado Mediterráneo Americano, mar interior en el continente americano compuesto por dos grandes lóbulos: el Golfo de México y el Mar Caribe. Su ubicación, lo conecta directamente con el litoral del este de México, y el sur de los Estados Unidos, siendo una comunicación privilegiada para la conectividad con el Atlántico, incluyendo Europa y el norte de África. Con el Caribe, si bien no constituye una localización directa, la fragmentación peninsular e insular de los espacios terrestres, facilita una relación expedita con América Central, las islas caribeñas, y el norte de América 
del Sur. Se incluye una situación privilegiada en relación con el Canal de Panamá, vía de comunicación, con todo el este de América el Sur, Asia y Oceanía. Evidentemente es una posición privilegiada de charnela, en un nodo comunicacional y de intercambio, que es el más privilegiado de todo el continente americano.

\section{Cuba y su Papel Protagónico en el Nuevo Regionalismo latinoamericano}

Desde el punto de vista geopolítico y geoeconómico, el puerto se encuentra bajo el impacto de la concurrencia de dos bloques regionales que se están formando. Por una parte los puertos mexicanos, estadounidenses, los centroamericanos y el propio Canal de Panamá, se involucran con la lógica neoliberal, y de dominio hegemónico, conformado por el nuevo atlantismo, el Arco del Pacifico, el TLCAN (Tratado de Libre Comercio de América del Norte), que no incorporan sistémicamente al puerto del Mariel en su dinámica comercial.

Cuba, por otra parte, tiene un papel protagónico en el nuevo regionalismo latinoamericano, que se está conformando con una alianza entre MERCOSUL, ALBA y PETRO CARIBE, nuevo bloque político económico que se está conformando con países de corte más progresista en una lógica más socio-estatal que se aleja de la visión neoliberal. Aquí Brasil, tiene un papel articulador, que se conecta a través de Venezuela por tierra, y el Atlántico con toda Meso América. Ese bloque, está conformando relaciones económicas y políticas con el BRICS, que incluye a China, India, Rusia y Sudáfrica. En general, si existen relaciones de carácter mercantil, un papel fundamental en esta vinculación la tiene la cooperación económica y política entre sus diversos integrantes, con un papel protagónico de la lógica estatal.

En realidad lo que está aconteciendo es la sustitución del modelo rígido de producción fordista en un Estado Nación de todo el conjunto de bienes y servicios, por un nuevo sistema pos fordista y globalizado, en el que se privilegia la producción de partes de productos o bienes y de determinados servicios que se conjugan con la manufactura en otros puntos, y dan lugar a la producción articulada de un bien, resultado de elaboraciones en diferentes lugares.

Se trata, de que en ese nuevo escenario va predominado la re localización de las plantas fabriles; la multi localización de la producción industrial; la tercerización sistemática y la consecuente formación de redes productivas que involucran un número mayor de actores socio-económicos y de escalas geográficas variadas movilizando un aparato tecnológico extremadamente sofisticado para viabilizar interacciones materiales (navíos porta-contenedores de última generación) e inmateriales (sistemas de telecomunicaciones de todo tipo. Son sistemas productivos organizados en redes: las industrias y los conjuntos productivos más flexibles que transforman progresivamente el espacio económico mundial en un gran conjunto de puntos especializados en la elaboración de las partes del todo. 
Se multiplican así, territorios productivos de diversos tipos alrededor del mundo: distritos de pequeñas empresas, polos tecnológicos, conjuntos productivos locales, zonas francas de grandes dimensiones o, plataformas logísticas En la actualidad, la interconexión de esos puntos y regiones especializadas propicia la formación pulverizada en unidades esparcidas por todo el mundo.

En la medida en que los intercambios internacionales de mercancías son preferencialmente realizadas por vía marítima, los puertos ocupan una posición estratégica en el seno de esa economía de circulación. En ese contexto los mega puertos van adquiriendo un nuevo papel al viabilizar la circulación de personas y de volúmenes gigantescos de mercancías, y se insertan en ese nuevo escenario como medios estratégicos y la conjunción de esas partes dispersas por el mundo.

En tales condiciones se privilegian factores de localización tales como el grado de profesionalización de la mano de obra, las condiciones de accesibilidad, la proximidad de un mercado de consumo de gran porte, la estabilidad institucional etc. Independientemente del contexto geopolítico de formación de bloques regionales y transnacionales, el puerto del Mariel se localiza en el corazón del espacio global de los flujos inherente a la economía de circulación de la globalización pos fordista contemporánea, de los circuitos comerciales globales y de la rutas marítimas internacionales. Es por ello que podrá constituirse en un nodo principal de articulación de redes productivas crecientemente globalizadas tomando el carácter de un "hub", o sea, un centro de procesamiento, distribución e integración de cadenas productivas fragmentadas internacionalmente. Sería de tal modo un puerto de la integración en una red territorial globalizada que se articularía a procesos globales y un punto estratégico de los grandes corredores marítimos.

Sin lugar a dudas, el desafío es buscar la manera, en que más que una confrontación, geopolítica y un escenario de competitividad acérrima se promueva una cooperación, entre los dos grandes bloques, y los diferentes Estados Nación que lo conforman, y el aprovechamiento de la situación y posición del fuerte en el nuevo espacio globalizando que cada día se amplia y se consolida más.

La complementariedad, la conectividad, y la articulación entre los bienes y servicios que se oferten, deberá basarse en una conjugación espacial y territorial. Las autoridades gubernamentales, emppresariasles, deben, partiendo de ese contexto ir a la búsqueda de negociaciones, que permitan el flujo de inversiones, de acuerdo a los requisitos que se exigen la creación de la ZED. Diagnosticar y pronosticar tendencias, y diseñar escenarios que propicien una planificación cuidadosa de la organización espacial y territorial, y contribuyan a la formación de una estrategia de negociaciones, será sin duda un reto en la producción del conocimiento.

Para Cuba, otro desafío de carácter geopolítico tiene que ver con las relaciones con los Estados Unidos. Ello pudiera constituir un salto de calidad, no solo en términos económicos, sino también políticos. Y es así porque se percibe este poten- 
cial engarzamiento como un incentivo para la gradual normalización de relaciones entre Cuba y los Estados Unidos. Una de las posibilidades que tiene Cuba de conseguir una normalización imprescindible de sus relaciones con los Estados Unidos es ofreciendo suficientes incentivos económicos. O sea aumentando los beneficios tangibles para ambas partes. Ello implica el enfrentamiento de determinados riesgos y costos políticos a los que Cuba está preparada a afrontar.

La ubicación geográfica del puerto favorece la llegada de buques desde los EEUU, sí se levantara el bloqueo, y permitiría para ambos países:

- Aumentar la diversificación de fuentes de ingreso.

- Incrementar la posibilidad de expandir negocios en América Central y el Caribe.

- La posibilidad de tener acceso al mercado de Estados Unidos por su cercanía geográfica.

- Generar 3000 empleos directos y 5000 indirectos.

- Favorecer la vinculación con la zona económica exclusiva de Cuba en aguas del Golfo de México.

- Potenciar nuevos mercados y constituir un polo de expansión y atracción del capital económico y tecnológico.

Brasil, tendrá debido a su protagonismo en la implementación del puerto y la Zona Especial de Desarrollo, un papel privilegiado, en el uso del mismo, con el propósito de incrementar su presencia en Meso América, acrecentar la conectividad y las relaciones a nivel macro regional y global, y constituirse en un interlocutor más creíble y confiable que promueve el progreso y las relaciones pacíficas, reforzando su papel de potencia macro regional.

\section{Impactos a Nivel Nacional}

Para Cuba, la construcción del puerto, desde el punto de vista territorial reporta fundamentalmente tres ventajas:

1. La desconcentración espacial, con la re funcionalización del puerto saturado en la bahía ambientalmente colapsada de La Habana.

2. El aumento de la conectividad e interrelación a nivel macro regional y global.

3. La movilización de potenciales de recursos y servicios naturales, económicos, y humanos en los diversos espacios y lugares del país. 
El occidente del país, con esta mega obra, incrementa su valor en todos los sentidos, y se reafirma como la macro región de mayor desarrollo del país. Redundará en una mayor articulación con los espacios económicos, en particular las regiones agrícolas y turísticas de Pinar del Río, y la parte central del occidente; con los espacios turísticos, y con los polos de desarrollo de Matanzas, Santa Cruz del Norte y Cienfuegos, entrelazados por óleo y gaso ductos. Promueve además el encadenamiento productivo y de valor agregado entre diversas actividades económicas en el occidente del país.

Sin embargo, es necesario tener en cuenta que la organización espacial que se re configure en el país, deberá estar marcada por los siguientes propósitos básicos:

- Garantizar el equilibrio espacial y territorial lo cual exigirá la disminución de las diferencias entre los centros y las periferias, tratando de limitar la configuración de espacios periféricos y marginales en torno a los enclaves, y las redes las conectividades que se está formando. Ello tiene que ver en lo fundamental, con la necesidad de consolidar un sistema de asentamientos urbanos, coherente, y espacial y paisajísticamente equilibrado.

- Limitar la concentración espacial económica, demográfica y política, conteniendo la sobre urbanización, la metropolización, no privilegiando la incidencia directa y autónoma de las redes globales y la migración anárquica.

- Fomentar un nuevo espacio reticular equilibrado, que conduzca a altos niveles de conectividad y de integración en todo el país, fomentando nodos y nexos que potencializan los flujos y una jerarquía racional.

- Asegurar una localización, distribución y organización de mercados de bienes y servicios regular en todo el país, evitando la hiper concentración económica, la segregación espacial, y el debilitamiento de los centros de convergencia, de servicios y espacios públicos.

- Propiciar el enraizamiento de la población expuesta, garantizando la oferta de empleos y oportunidades, de tal manera que se consolide una identidad territorial coherente, y diversas maneras de asegurar la equidad e inclusión socio espacial.

Quizás, el propósito más importante será el de lograr un alto nivel de interacción entre el equipamiento técnico e innovador, y la estructura empresarial de la ZED, y toda su hinterlandia, conformada por la estructura regional y territorial que le sirve de soporte. En concreto estos objetivos deberán propiciar, que disminuya la desigualdad regional, y en particular la diferencia con las regiones orientales. Se trata de asegurar la descentralización, la des concentración y la existencia de formas de crecimiento espacial difuso. 
El hecho de la existencia de infraestructuras de conectividad, y la limitada presencia de obstáculos y barreras espaciales para la conjunción entre los espacios en todo el país, debe constituir un estímulo para el logro de este propósito. Es evidente, que se impone el desafío de conocer a manera en que puedan establecerse las complementariedades, la difusión de os beneficios de la construcción de la mega obra, con la creación de clusters espaciales y cadenas de encadenamiento dl valor agregado, apoyados en una matriz y un soporte espacial y ambiental coherente. Estas continuidades espaciales, deberán ser motivos de trabajos específicos de diagnóstico y de diseño de procesos de ordenamiento territorial, sustentados en la ordenación ambiental.

\section{Impactos a Nivel Regional}

La creación del puerto y la ZED de Mariel inducirá a la potenciación de una región geográfica, un verdadero "hinterland "que deberá abarca en lo fundamental la provincia de Artemisa, aunque podrá alcanzar porciones de las provincias de Pinar del Rio, La Habana, e incluso Mayabeque. Esa región deberá sobre pasar con creces el área dedicada a la zona especial de desarrollo, y estará condicionada por el poder de atracción y de incidencia, que actuará como un campo de fuerzas relevante.

La organización espacial de la región deberá incluir los siguientes espacios:

- del sistema portuario: ubicado en las inmediaciones de la instalación de las consunciones portuarias, que incluye además algunas edificaciones fabriles, instalaciones petroleras y administrativas, zona de protección ambiental, y asentamiento urbano;

- de la difusión industrial: presumiblemente, luego de la expansión del desarrollo industrial en el espacio portuario, es posible su localización en un radio de unos 10 kilómetros a partir del puerto; abarcará, el área del actual desarrollo industrial, situada en el borde oriental dela bahía, la cual conformó un mini polo de desarrollo, afectado por la localización en una estrecha faja, en el borde inclinado de una meseta, sometida a deslizamientos, y el crecimiento irregular de edificaciones urbanas y de expansión informal; la situación ambiental critica de esa franja, exige de su reubicación y mejoramiento;

- de explotación agrícola: pueden distinguirse dos sub espacios - el septentrional, localizado en una llanura ondulada y colinosa, de potenciales medios y bajos, actualmente muy poco aprovechado, en el que predominan potreros poco productivos, matorrales y restos de bosques secundarios; con un ancho máximo de 15-20 kilómetros, se combina con el área de desarrollo industrial 
potencial; el meridional, formado por la llanura calcárea de suelos rojos ferralíticos, de alto potencial agrícola y ata productividad; la misma se explota con alta intensidad, básicamente para la producción de tubérculos, hortalizas, y frutales, que en la actualidad abastecen en lo fundamental a la capital del país; existen sin embargo, algunos vacíos espaciales, influenciados por procesos erosivos y de carsificación, y grandes reservas de explotación del potencial;

- de desarrollo turístico del litoral: a unos 30 kilómetros en la dirección tanto oriental (hasta la bahía de Cabañas) y occidental (hasta el poblado de Baracoa, en el extremo este de la provincia Artemisa), se extiende una llanura litoral formada por una cuesta calcárea baja (altura máxima de 50 metros), con hasta tres niveles de terrazas marinas, relativamente seca, sin potencial agrícola; en la misma se localizan pequeños abrigos formados por playas estrechas de concha; la asimilación turística e incluso urbanística es muy limitada, con un potencial relativamente alto para ambas actividades;

- de protección de la naturaleza y desarrollo turístico: a unos 30 kilómetros en dirección oeste se localiza en la cadena de alturas y montañas bajas estructuro erosivas y parcialmente carsificada y boscosa de la Sierra del Rosario, la reserva de la biosfera del mismo nombre, una de las seis actualmente existentes en el país; constituye un área de protección y de turismo ecológico de renombre internacional, y será sin dudas un atractivo complementario a la zona de desarrollo;

- de protección de la naturaleza: en la llanura litoral, en varias colinas y pequeñas alturas de la llanura calinosa, y en el borde inclinado limítrofe con la llanura roja, será necesario la instalación de espacios de protección ambiental que deberán garantizar en lo fundamental el funcionamiento de las cuencas que vierten a la bahía, y la manutención de los nacimientos de cañadas y arroyos.

\section{El Sistema de Asentamientos}

En la actualidad el sistema de asentamientos se subdivide en dos espacios. El sub espacio septentrional, tiene como centro al poblado del Mariel. Tiene un carácter disperso y se forma por pequeños poblados básicamente rurales, entre los que destaca Quiebra Hacha, que es el punto de entrada actual del puerto. El sub espacio meridional , formado por una red de ciudades pequeñas del hinterland agrícola, que tiene a Artemisa (capital provincial) y a Guanajay, en una situación centro oriental como centros jerárquicamente sobresalientes. 
En particular este último, deberá tener un papel muy importante como conmutador espacial, ya que en sus inmediaciones se producirá la conexión entre la conectividad meridional que parte del puerto con la ciudad de La Habana y el resto del país. Se presume un rápido e intenso crecimiento urbano en los cuatro puntos mencionados, que exigirá de un re acondicionamiento urbanístico, un mejoramiento ambiental, y la protección y re calificación del paisaje urbano, para garantizar lugares con identidad propia,con valores patrimoniales conservados y que puedan ser apropiados socialmente.

\section{El Sistema de Conectividad}

En la actualidad se forma por dos tipos de ejes: los latitudinales y los meridionales. Los primeros conjugan diversos puntos de la región entre Pinar del Rio y la Habana. El eje septentrional, pasa por las cercanías del puerto, y entronca a Mariel con la capital nacional. El segundo situado unos 20-30 kilómetros al sur, se forma por una autopista moderna y la antigua carretera central (que data de unos 80 años). La conectividad sub meridional entronca los poblados y ciudades del sistema urbano septentrional con el meridional, en particular con Artemisa y Mariel. A excepción de la autopista a Pinar del Rio, toda esa red de conectividades, exige de su remodelación y mejoramiento. En la actualidad se construye un vial que conectará al puerto con la Ciudad de la Habana, en dirección sub meridional para entroncar con el eje latitudinal. Será necesario precisar el carácter que tomara la vía panamericana, que entronca Mariel con La Habana, y que tiene un inmenso potencial de carácter turístico y urbanístico. La puesta en valor y la producción de esos espacios, en la actualidad débilmente conformados y explotados, exigirá de un intenso pero cuidadoso proceso de re ocupación, re asimilación y re apropiación de espacios y recursos.

Ese proceso, puede estar sujeto a dos tipos de posibles impactos:

- El vinculado con la acción de empresas trans nacionales, y los grandes grupos empresariales internacionales que se caracterizan por conformar un direccionamiento externo, de las actividades productivas regionales. Así, la empresa no controla solo el aparato de la producción sino que controla sus mercados y toda la estructura física (o virtual) articulada con la comercialización y los servicios involucrados. Como agentes económicos territorializados, tales empresas pueden generar situaciones de sobre utilización de los potenciales naturales y físicos y de vacíos económicos, debido a procesos de re estructuración sectorial, llevando a ciudades y regiones a situaciones de inestabilidad y crisis económicas.

- El vinculado con la acción de empresas públicas, cooperativas o privadas, que motivado por el afán de obtener ganancias a corto plazo y a todo costo, 
se dirigen a la ocupación irregular del espacio, a la no observancia de la organización espacial natural, y a la intensificación del uso, provocando inestabilidades.

En ambos casos se producen verdaderas tensiones ambientales, espaciales y territoriales . Las empresas pueden ser entendidas bajo la óptica territorial en la medida en que actúan en determinadas áreas, construyen sus "territorios", no solo actuando de forma regionalizada, sino organizándose en redes donde establecen también una parcela de la división del trabajo, colaborando para el arreglo territorial (local y regional) y para la definición (temporal) de una determinada dinámica social, que resulta en una acción en el espacio.

En ambos casos se puede generar modelos espaciales desarticulados caracterizados por:

- La degradación del soporte físico-natural, de la estructura, y funcionamiento de los sistemas naturales y de la pérdida de recursos y servicios ambientales.

- La pérdida de los potenciales de servicios y de recursos, y el deterioro de los valores agregados del espacio económico.

- La desestructuración del sentido de pertenencia territorial de los actores y agentes; y la desapropiación del espacio social por parte de la población local.

- La alteración de la identidad y la imagen territorial: y del entorno simbólico, y la pérdida del patrimonio paisajístico del paisaje cultural. Evitar el surgimiento de las anomalías, exigirá por parte de las autoridades, en la formación de la Región Polarizada del Mariel, de un esmerado trabajo de planificación y puesta en marcha un programa de ordenamiento ambiental y territorial. Las mismas deberán regular la formación de la organización espacial que implica, el uso y ocupación ordenada de un espacio apropiado socialmente, y la cohabitación o sea, la convivencia espacial de los seres humanos.

Para el logro de ese propósito, será fundamental establecer y consolidar la gobernabilidad territorial, en la que el Estado refuerce su poder hegemónico. Ello deberá lograrse concibiendo al territorio, como espacios cohesionados, conectados y con identidad propia, en el que en el contexto de múltiples territorialidades conformadas por un conjunto e actores sociales y agentes económicas. En ello la autoridad estatal ejerce un papel protagónico de regulación institucional, de uso y transformación de los recursos como instrumento de poder y de gobernanza activa, en aras de los intereses de la nación.

Ese proceso de re estructuración ambiental, espacial y territorial, exige, además de medidas institucionales, y de elaboraciones técnicas, basadas en procedimientos taticos y operativos, de la obtención de conocimientos, que precisen establecer, y 
diagnosticar todas las complejidades actuales y futuras, sustentadas en una fundamentación teórico conceptual coherente, y en la obtención de datos e informaciones empíricas. Deberán obtenerse conocimientos sobre las propiedades y atributos y las tendencias dinámicas y evolutivas, y las reacciones y respuestas a las diferentes acciones del espacio natural, del económico, el social y el cultural. En particular la integración cognitiva al nivel territorial, exigirá de considerar la dialéctica entre el espacio geográfico como un espacio de poder, y como un soporte y una matriz y marca espacial.

\section{Impactos a Nivel Local}

Este nivel tiene que ver con el sitio de emplazamiento del puerto, en la Bahía del Morel, bordeada por una cuesta calcárea litoral, ya la que vierten las cuencas de los pequeños ríos Bango (o San Miguel) y Cañas. Desde el punto el vista político administrativo el nivel local se representa por tres de los cinco conejos populares que forman parte del Municipio del Mariel: los de Quiebra Hacha, por el oeste, y los de Mariel y La Boca por el este.

El elemento central de todo el proyecto del puerto del Mariel es la bahía del mismo nombre. La misma es una bahía de bolsa, de unos $6 \mathrm{kms}$ de largo en dirección sub meridional, de largo mínimo en el canal de entrada de unos 500 metros, y ancho máximo en su porción central de unos $4 \mathrm{kms}$. Su profundidad máxima de unos 10 metros.

La bahía forma un geosistema para genético con las cuencas de Bango, de unos 30 kilómetros cuadrados y la del Cañas de unos 10 kilómetros cuadrados. Son cuencas de altura máxima de 100 metros, formadas en rocas volcánicas y flish, y margas, constituidas por colinas bajas y terrazas aluviales encajadas. Toda la bahía prácticamente es bordeada por costas bajas pantanosas con bosques de mangle.

La mayor vulnerabilidad de la bahía es su posible sedimentación por depósitos provenientes de las mencionadas cuencas, y la posible contaminación, por las infraestructuras que se instalen. En la actualidad, a pesar e la existencia de instalaciones en la faja oriental, y del desarrollo urbano de La Boca - Mariel, el estado de las aguas es satisfactorio. Por lo visto, los desbrozes, y los rellenos y destapes producidos para la construcción de los puertos, no ha dado lugar a procesos negativos significativos. El emplazamiento del puerto, en un espigón margoso situado al borde de un pequeño lóbulo septentrional, cercano a las canal, da lugar a que dichos depósitos son acarreados con rapidez al mar abierto.

No obstante, teniendo en cuenta la circulación semi cerrada de las corrientes en la bahía, se hace imprescindible prestar un cuidado especial a través de un diseño ambientalmente concebido de la zona portuaria, del distrito industrial y los 
emplazamientos asociados a la mega obra. También es imprescindible, prestar una atención esmerada a la ocupación de los espacios de la llanura ondulada en las cuencas, con el propósito de minimizar los posibles impactos negativos, reducir la eutrofización y la acumulación de depósitos en la bahía, lo que exigirá de procesos especiales de diseño ambiental.

Se torna imprescindible, el diseño y la implantación de un sistema de áreas protegidas, a escala municipal, que abarque, los sistemas ambientales cuasi naturales de la cuesta que bordea por el norte la bahía, los bosques costeros, y las colinas y vertientes más vulnerables de las cuencas.

Constituye un desafío para potenciar el nivel local, asegurar que el que sistema regional que se forme con la implantación del mega proyecto del puerto, no adquiera un carácter centro periférico, sino que se caractericen por ser sistemas multi polarizados no jerarquizados. Para ello, es fundamental, salir de la situación de relativo abandono y atraso, de la mayor parte de los asentamientos humanos, que conforman los consejos populares involucrados. Los mismos, tienen serios problemas del estado de las viviendas y las infraestructuras, estructuras urbanos deficientes, carencia de área verdes, lugares de encuentro y espacios públicos, red vial deteriorada tanto en los asentamientos como a nivel municipal; serios problemas de contaminación de los ríos, carencia de acueductos y servicio de alcantarillados, serios problemas con la valorización del patrimonio paisajístico y urbanístico; y falta de servicios principalmente gastronómicas.

Revertir esa situación, exige de la puesta en marcha de proyectos a nivel local. Para ello, se necesita de empoderar a la población local, otorgándosele prerrogativad para la implantación de proyectos, en los que prime una participación y gestión activa. Para ello debe estimularse la organización de cooperativas agropecuarias y no agropecuarias, pequeñas y medianas empresas, y el fomento de la pequeña iniciativa privada (cuenta propistas). Para ello se exige de un conocimiento riguroso, de los elementos que potencian el nivel local, en particular el inventario y diagnóstico de la confirmación de os lugares (sus espacios vítales, productivos, sociales y culturales), las acciones y la gobernabilidad territorial, las potencialidades, la caracterización de as comunidades, y de la cultura local, bloqueos y limitantes en el nivel local, la asimilación de tecnologías apropiadas.

Es fundamental para ello, que pate de los lucros y ganancias de la implantación del puerto y sus instalaciones se revierta al nivel local, y la población pueda tener una participación activa en el uso de los recursos, con el propósito de optimizar el nivel y la calidad de vida de los pobladores.

\section{Impacto de los Procesos de Desarrollo}

El mega proyecto del Mariel, genera sin dudas procesos de desarrollo a todas las escalas espaciales. Ello tiene que ver con los tres componentes fundamentales en 
el proceso de desarrollo: 1) El componente del crecimiento económico, dado por el incremento en la producción de bienes y la satisfacción de servicios, como resultado de la instalación de un capital físico tangible. 2) El desarrollo, considerado como el traspaso de los beneficios del crecimiento en satisfacer las necesidades básicas de la población, y en el incremento del nivel y la calidad de vida. 3) La optimización, e incremento de la complejidad funcional, el mejoramiento de las estructuras, de las relaciones sistémicas, en el interior de los territorios, considerados como portadores del proceso de desarrollo.

En este sentido se define al desarrollo territorial como el proceso de de articulación de las estructuras políticas, sociales, económicas y ambientales de territorios determinados (un país o región, Provincias, municipios), para la movilización más completa de sus potencialidades, mediante procesos relacionados con el alcance de ciertos propósitos (igualdad, crecimiento, sustentabilidad) que tengan el objetivo de garantizar el bienestar de su población.

A nivel nacional, la mega obra, permitirá incrementar la posición de Cuba al nivel económico y comercial internacional; abrir todo el quehacer económico y social del país a la asimilación de nuevas tecnologías; e incrementar la explotación de os recursos de todo tipo. Fomentará, la conectividad entre diversos espacios y territorios e incrementará el nivel de desarrollo da varias regiones del país. Al nivel regional (teniendo en cuenta para ello la provincia de Artemisa, y partes que constituyen el campo de fuerza de la mega obra en las provincias de Pinar del Rio, La Habana y Mayabeque), contribuirá a los procesos de desarrollo regional y territorial.

En este sentido es necesario precisar las diferencias entre los conceptos de región y territorio, para esclarecer que se entiende por el desarrollo en esas dos categorías. La región es el resultado de proceso de individualización, a través de la articulación, diferenciación y estructuración del espacio, teniendo una connotación realista relacionada a las prácticas sociales como hecho, siendo la evidencia concreta de la diferenciación espacial. Es el resultado de la evolución de la humanización de la superficie terrestre, en la que se conforma una determinada singularidad y personalidad.

Se distinguen dos tipos fundamentales de región: la homogénea y la polarizada o nodal. El desarrollo regional, tiene que ver con el proceso de desarrollo de manera evolutiva, y como resultado de la articulación espacial en un nivel individual. La región homogénea, se caracteriza por una determinada estructura espacial, con características relativamente similares. La polarizada se define a partir de la interdependencia funcional y la densidad de flujos entre sus elementos, organizándose un sistema espacial en torno a un centro o nodo, constituyendo su campo de influencia. El territorio, es el producto de una intervención consciente, vinculada a una determinada estructura del poder, de espacios concretos. Constituye además 
un instrumento de control, producción y significación del espacio.

El territorio estatal, es el resultado de la conformación político administrativa, que generalmente comporta en su interior diferentes territorialidades, que son espacios de poder de diferentes agentes económicos y actores sociales.

El desarrollo territorial, es aquel proceso inducido de manera directa,(a través de determinados programas) por las fuerzas de poder, generalmente teniendo al Estado como elemento facilitador o incluso propulsor.

Sin dudas, existe una relación entre región y territorio. El territorio, generalmente se vincula a una determinada estructura regional, existiendo una dialéctica entre región y territorio, que es necesario entender para estudiar y estimular determinados procesos de desarrollo. Tanto en el desarrollo regional como territorial, un papel esencial lo desempeña la innovación, como una vertiente que impulsa nuevos horizontes, teniendo al conocimiento como brújula fundamental y que conduce a la transformación constante de las regiones.

Este desafío considera como fundamental al sistema de innovación, que caracteriza por una conjunción de instituciones públicas y privadas que interfieren en los aspectos económicos, sociales y geopolíticos de una región, por el desarrollo y la formación de nuevas estructuras tecnológicas, lo que propicia la creación de nuevos productos o procesos. La innovación ocurre no solo en las empresas sino prácticamente en todo el quehacer social a través de políticas gubernamentales, leyes, identidades culturales, normas técnicas y aspectos sociales, que tienen que ver con el consumo, el financiamiento y la regulación tecnológica utilizando a los agentes que tiene que ver con los conocimientos científicos y tecnológicos (universidades, centros de investigación, entidades financiadoras y otras instituciones).

En este sentido, la creación de polos de modernización tecnológica, como política estructurante a nivel territorial, como vector del desarrollo regional y territorial y como nodo de innovación, permite considerar a la ciencia y la tecnología como componentes de la competitividad sistémica. Tanto las regiones como los territorios se conforman por espacios productivos.

Los mismos pueden clasificarse en tres tipos a partir de la perspectiva de la existencia de un proceso de innovación: 1) Espacio productivo desarrollado: ocurre la caracterización de un escenario perspectivo de investigaciones avanzadas, nuevos procesos informacionales y proyectos con productos y servicios de punta. 2) Espacio productivo en desarrollo: caracterizado por la acción de investigaciones iniciales, desarrollo gradual de procesos informacionales, y de sub proyectos de productos y servicios, en fase inicial de análisis. 3)Espacio productivo periférico: se caracteriza por pequeñas o ninguna acción de las investigaciones, débil proceso informacional, y poca o ninguna acción de proyectos y servicios.

Mariel se encuentra enclavado en la región geográfica de La Habana-Matanzas, en particular en la sub región del la Llanura del Norte. Está en los límites de 
las regiones de la Llanura del Norte de Pinar del Río y de Artemisa. Todas estas regiones, en particular las dos primeras se caracterizan por una baja ocupación y asimilación espacial, un desarrollo económico limitado, y espacios productivos con niveles de innovación muy débiles. Artemisa, al constituir una región de intensa explotación agrícola, tiene un desarrollo económico y de innovación intermedio. La construcción de la mega obra de Mariel, podrá sin dudas, dar lugar a la creación de una región polarizada de fuerte incidencia en todo el país. El nivel de innovación deberá ser muy alto. Su incidencia deberá afectar toda la región de la llanura norte de la Habana, el norte de Pinar del rio, e incluso parte de la región urbana de La Habana, y el norte de la región de Artemisa.

Sin embargo, es presumible, que esa polarización de lugar a vacíos espaciales, al relativo estancamiento de regiones colindantes, y un carácter de dependencia de regiones contiguas, dando lugar al traspaso de recursos (humanos, materiales) de otras regiones hacia esa nueva región funcional. Por lo tanto, los posibles efectos negativos de un presumible desarrollo polarizado y anómalo, deberá ser compensado, pero sobre todo corregidos y regulados por el desarrollo territorial. Cabe, en primer lugar a la provincia de Artemisa, en articulación en particular con las autoridades de la provincia de La Habana, de tomar las medidas, para asegurar un equilibrio territorial. La función de planificación, regulación e incluso difusión de los procesos de innovación deberá sustentarse en soportes espaciales de articulación y conectividad, y deben estar sustentados en herramientas de intervención gubernamental. Se trata por tanto de obtener conocimientos sobre las tendencias y las acciones de desarrollo, para encauzar las políticas y decisiones a tomar.

En cuanto al desarrollo local, no hay otra opción que promover procesos participativos y conscientes de apropiación de los lugares por parte de la población local. Hay que priorizar el empoderamiento de las comunidades para el uso racional de los recursos locales, promoviendo la endogenización, y el traspaso de beneficios de la explotación de la zona especial de desarrollo del Mariel a esos propósitos. Teniendo en cuenta, las condiciones en que en Cuba se promueve el desarrollo local, será imperativa una participación protagónica de las autoridades y empresas estatales, aunque dando un margen de acción a las comunidades locales. Todo ello, exige de un conocimiento riguroso del contexto en que se desarrollan esos procesos. Todos estos procesos de desarrollo que se implementen, precisarán sin dudas de incorporar a la sostenibilidad como elemento fundamental. En realidad el Desarrollo Sostenible, implica la articulación de tres patrones de sostenibilidad, inherentes a los sistemas que se encuentran en un determinado territorio: el económico, el social y el ambiental. La concepción de Desarrollo Sostenible, en su interpretación original y clásica, estuvo fuertemente sustentada en el ambientalismo naturalista. De tal manera, se considera como, que en el Desarrollo Sostenible los sistemas económicos y sociales, debían de subordinarse o adaptarse a las características de 
los sistemas naturales.

El diseño de escenarios en que la sostenibilidad se incorpore a los procesos e desarrollo, exigirá de conocimientos profundos sobre la sostenibilidad de los diferentes sistemas, y las formas en que la misma deberá adecuarse en las diferentes regiones y territorios.

\section{Consideraciones Finales}

En la actualidad, se vive una etapa en que es necesario repensar, redescubrir valores y reverenciar momentos y de comprender desafíos que han sido hasta ahora desconocidos y complejos. El impacto ampliamente abarcador y a todos os niveles territoriales de la implantación de la ZED del Mariel, exige, que todo lo relacionado con los procesos de desarrollo y las transformaciones espaciales y territoriales que generará, no puede dejarse a la espontaneidad.

Solo el conocimiento apoyado en el saber científico, en la ciencia como institución social, y en una sólida fundamentación teórico conceptual y metodológica, podrá brindar los datos, las informaciones, y las reflexiones que permitan con certeza trazar los rumbos necesarios para tratar de enrumbar los procesos de desarrollo. El espacio geográfico, el territorio y el medio ambiente constituyen la base, el fundamento para cualquier proceso de desarrollo. Vivimos en el medio ambiente y del medio ambiente. Por lo tanto, conocer el medio ambiente es un requisito fundamental para la construcción de cualquier proceso de sociedad del conocimiento. La centralidad del conocimiento lo convierte en pilar fundamental de la riqueza y el poder de las naciones, siendo la misma función de la gente, del cultivo de la inteligencia del pueblo. Es por ello que se plantea la necesidad de priorizar la inversión en el capital humano, o sea, en educación, e información.

En ese sentido se hace imprescindible un dialogo constante entre la ciencia, las empresas, los tomadores de decisiones y la población local, para que se puede de la manera más abarcadora asimilar en toda su complejidad las transformaciones de todo tipo y a todos los niveles que ocurrirán como resultado de la implantación de la ZED del Mariel. Una educación para la sostenibilidad, exigirá de un esfuerzo fundamental en socializar a todos los niveles el conocimiento que la ciencia podrá producir.

El gobierno cubano ha designado al Instituto de Planificación Física (IPF) la responsabilidad de elaborar el Ordenamiento Territorial que deberá regir en la implantación de la ZED. No obstante, los conocimientos que exige la implementación de esa mega obra, rebasan los marcos técnicos y regulatorios de ese instrumento de planificación.

Teniendo en cuenta la necesidad de garantizar la obtención de conocimientos científicamente fundamentados, la alianza CBS ha orientado la elaboración de un 
proyecto de investigación que se dirija en lo fundamental a determinar los impactos de la construcción del puerto y la implantación de la ZED del Mariel, desde una perspectiva espacial, territorial y ambiental, y en el contexto de los procesos de desarrollo que generará dicha mega obra. Los resultados de dicho proyecto podrán constituir una base fiable para la elaboración de proyectos de ordenamiento y de gestión ambiental y territorial .

El proyecto ha sido elaborado por un equipo conjunto cubano brasileiro en el marco de alianza CBS y en la colaboración entre las Academias de Ciencias de Cuba y Brasil.

El equipo cubano es coordinado por la Facultad de Geografía de la Universidad de La Habana, y lo integran representantes de diversos centros de investigación, del IPF, y de las autoridades y empresas que laboran en el puerto. Dicho equipo aportará la información básica, y la vasta experiencia en la esfera de la planificación y la gestión ambiental y territorial.

El equipo brasileiro es coordinado por el Departamento de Geografía de la Universidad de Campinas, en el Estado de Sao Paulo. Está integrado por representantes de varias universidades brasileiras, de entidades gubernamentales y estaduales y de empresas gestoras de puertos. El equipo aportará la experiencia en la valoración y construcción de puertos en Brasil, y la vasta experiencia en la formulación teórico metodológica de los problemas del desarrollo regional y local. De este modo, la colaboración entre científicos y técnicos de Cuba y Brasil, pone a disposición de los gobiernos y la sociedad de sus respectivos países, un esfuerzo concreto en la aplicabilidad práctica de la construcción de una sociedad del conocimiento, marcada por el interés en colocarla a disposición de las necesidades de la población y de proyectos nacionales y regionales de alcance social, y no del poder hegemónico mundial.

\section{Referencias}

LIMA Luiz Cruz, MONIÉ Frédéric, BATISTA Francisca Gonçalves - A nova geografia econômica mundial e a emergência de um novo sistema portuário no Estado do Ceará: o Porto do Pecém; Terra Livre Goiânia Ano 20, v. 2, n. 23 p. 97-109 Jul-Dez/2004

MATEO RODRÍGUEZ, José - La dimensión espacial del desarrollo sostenible: una visión desde América Latina., Editorial U.H., Editorial Científico Técnica, La Habana, 2012, 293 pgs.

MONIE, Frédéric - Dinâmicas produtivas, logística e desenvolvimento territorial., Em: VIDEIRA, S.L., COSTA, P.A., FAJARDO, S. (Orgs.).- "Olhares geográficos: modos de ver e viver o espaço, Rio de Janeiro, Bertrand Brasil, 2012, pp. $145-168$.

Recife, Olinda, Maceió, 2014 